\title{
Spectral/cepstral analyses of connected speech in parkinson's disease as compared with sustained phonation before and after voice treatment
}

\author{
Ghadah G. Alharbi', Michael P. Cannito², Eugene H. Buder', Shaheen N. Awan ${ }^{4}$ \\ ${ }^{1}$ Department of Special Education, University of Jeddah, Jeddah, Saudi Arabia; ${ }^{2}$ Department of Communicative Disorders, University of Louisiana, \\ Lafayette; ${ }^{3}$ School of Communication Sciences and Disorders, University of Memphis, Memphis, ${ }^{4}$ Department of Communication Sciences \& \\ Disorders, Bloomsburg University of Pennsylvania, Bloomsburg, United States
}

Purpose: The primary purpose of this study was to assess the effect of Lee Silverman Voice Treatment (LSVT ${ }^{\circledR}$ LOUD) on selected spectral/cepstral measures of voice in connected speech. Spectral/cepstral analyses also were used to descriptively compare changes in connected speech to those previously reported in sustained vowels. An additional goal was to examine individual differences in responses to LSVT across the spectral/cepstral measures.

Methods: Nine adult participants with PD were examined in a pre/post treatment design. Speech recordings were obtained on three different days within one week before and one week after LSVT. Speech recordings were analyzed for cepstral peak prominence (CPP), CPP Standard Deviation (CPP-SD), Low/High Spectral Ratio (L/H SR), and Cepstral/Spectral Index of Dysphonia (CSID) using the Analysis of Dysphonia in Speech and Voice (ADSV) program.

Results: CPP and CSID were the only measures that detect treatment-related changes in connected speech. Investigating individual differences demonstrated more participants exhibiting an improvement in sustained phonation than in connected speech.

Conclusions: Cepstral/spectral measures have been shown to be valid measures for estimating dysphonia severity in both continuous speech and sustained vowels. In addition, it ex tends the use of the cepstral/spectral measures for characterizing speech and voice aspects prior to treatment and for quantifying treatment outcomes. Moreover, it supports the use of LSVT as a treatment approach for improving voice quality in addition to intensity in PD.

Keywords: Parkinson's Disease, Connected Speech, Voice Cepstral Analysis, Spectral Analysis

\section{INTRODUCTION}

Parkinson's Disease (PD) is an idiopathic neurodegenerative disorder that affects approximately one million Americans with 60,000 new cases diagnosed annually [1]. PD is characterized by classic motor symptoms of slowness of movement, muscular rigidity, postural instability and resting tremor [2]. In addition, reduced movement amplitude as seen in micrographia, mask-like faces, gait disturbances, dysphagia, and a variety of cognitive communicative disorders are common in individuals with PD. Of particular relevance for speech-language pathologists are disturbances of speech and voice which are likely to develop in nearly 90 percent of individuals with PD over the

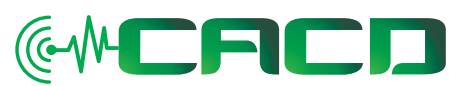

Received: May 13, 2021

Revision: August 30, 2021

Accepted: August 30, 2021

Correspondence:

Ghadah G. Alharbi

University of Jeddah, College of Education, Department of Special Education, 3715 Umm Khansar, AlRehab, Jeddah 23344, Saudi Arabia Tel: +966552986645

E-mail: ggalharbi@uj.edu.sa

(C) 2021 The Korean Association of SpeechLanguage Pathologists

This is an Open Access article distributed under the terms of the Creative Commons Attribution NonCommercial License (https://creativecommons.org/ licenses/by-nc/4.0/) which permits unrestricted noncommercial use, distribution, and reproduction in any medium, provided the original work is properly cited. 
course of illness [3]. Speech disorders of PD, termed hypokinetic dysarthria, are characterized perceptually by reduced intelligibility, decreased loudness, breathy voice, monoloudness, monopitch, imprecise articulation and disordered speaking rate $[4,5]$.

Respiratory and phonatory deficits in a person with PD affect the production of speech, leading to problems with phrasing and intensity [6]. Respiratory deficits may cause a reduction in vital capacity, a reduction in intraoral air pressure during consonant/vowel productions, and abnormal airflow [7]. Alternatively, it has been suggested that abnormal movements of both vocal folds (VFs) and the supra-laryngeal articulators may lead to atypical variation in airflow resistance, which may explain apparent abnormalities in respiratory function [7]. At the laryngeal level, hypo-adduction of the VFs, bowing of the VFs, supraglottic tremor and/or hyper function, and tremulous movements of the arytenoid cartilage have been demonstrated endoscopically in individuals with PD [8].

Studies investigating listener impressions of speech and voice characteristics in PD include decreased vocal intensity, decreased articulatory precision, mono-pitch, breathy or harsh voice quality in speech tasks [9], and less spectrally distinct consonants [10]. These are important factors affecting intelligibility $[11,12]$. Speech production studies in speakers with PD have demonstrated deficits in oral closure for stop consonants, reduced range of articulator motion for diadochokinetic (DDK) tasks, deficits in the amplitude and velocity of lip and mandible movements, and slowed articulator movement during vowel production and during speech tasks including oral reading of the first paragraph of the Rainbow Passage $[13,14]$. It has been reported that speakers with PD tend to inappropriately produce acoustic energy during the stop gap of voiceless consonant [15]. In addition, slowness of articulation was indicated by a shallower second formant slope during speech tasks including word reading [16]. At the supra-segmental level, more pauses and increases in fundamental frequency $\left(\mathrm{F}_{0}\right)$ with a reduction in its variability, reduced sound pressure levels (SPL), and rate abnormality have also been reported [10].

While some beneficial effects of levodopa on vocal performance have been reported [17,18], various PD therapies such as pharmacological or surgical speech interventions have not been shown to be effective in improving speech and voice quality of patients $[19,20]$ as compared to intensive voice treatments such as the Lee Silverman Voice Treatment developed for PD (LSVT ${ }^{\circledR}$ LOUD, or "LSVT"). LSVT is an intensive behavioral voice treatment developed for idiopathic PD [19], and has been shown to produce long-term voice improvements in speakers with PD lasting up to two years following treatment $[21,22]$. The effectiveness of LSVT has been demonstrated across three randomized blind control studies [21,2325] and a delayed treatment onset study [19]. Increased vocal intensity is the principal effect resulting from LSVT [23]. Additionally, other changes identified after LSVT including significant increases in the maximum duration of sustained vowel phonation, maximum fundamental frequency, habitual fundamental frequency and fundamental frequency variability in speech [23]. LSVT also demonstrates positive changes in perceived voice quality [11], acoustic harmonic structure of sustained vowels [26] and intelligibility posttreatment in speakers with PD [27]. Clinically, LSVT is a known intervention method which aims to allow the patient to habitually use a louder and better-quality voice in daily life $[23,25,28,29]$.

At the laryngeal level, a significant improvement in VF closure has been observed endoscopically in patients after undergoing LSVT treatment [30]. Additionally, decreases in electroglottographic open quotient accompanied by increases in subglottal pressure as well as rate of airflow shut off have been observed post-LSVT [31]. Such changes are consistent with the increased dominance of harmonics observed by Alharbi et al. [26] in the acoustic spectrum during voicing in sustained phonation tasks.

In voice quality assessments, it is important to assess both connected speech and sustained vowels. Several reports have found that measurements obtained from connected speech in certain populations may better predict voice classifications such as age and gender, differentiation of adductor spasmodic dysphonia from muscle tension dysphonia, and voice change pre- vs. post-thyroidectomy [32] than measurements obtained from sustained vowels [33-35]. It is important to assess both connected speech and sustained vowels because voicing behaviors differ for these tasks and because they assess very different dynamic vs. relatively static motor tasks respectively. Sustained vowels provide a relatively clear window into the balance between noise and periodicity related to glottal function or dysfunction without the potentially obscuring influences of laryngeal and supra-laryngeal articulation and prosodic variations [36-38]. However, connected speech provides the clinician with information on how the patient is able to rapidly vary laryngeal and supra-laryngeal postures and various prosodic vocal parameters for communicative purposes [39]. 
It has been reported that acoustic measures such as cepstral peak prominence (CPP) obtained from connected speech samples may correlate better with the perception of dysphonia severity than do sustained vowels [40]. In addition, voice disordered individuals with may produce variation and increased severity of dysphonia in typically longer duration connected speech passages versus dysphonia observed during relatively short duration segments of sustained vowel production [38]. Thus, both tasks may be considered equally important to consider in assessment and evaluation of therapeutic outcomes.

Acoustic measures may be classified into two broad categories; these are spectral/cepstral measures and traditional time-based acoustic periodicity perturbation measures. Accurate identification of cycle boundaries to determine $\mathrm{F}_{\mathrm{o}}$ is needed in order to make traditional time-based acoustic measurements of phonatory perturbation. Such measures include jitter, shimmer and some algorithms for harmonic-tonoise ratios. If dysphonia is present resulting in an already unclear or perturbed periodicity of the voice, then the onsets and offsets of cycles will be particularly difficult to identify [41]. Thus, traditional time-based acoustic measures may not be appropriate for the analysis of PD due to the acoustical variability, dysphonia, and subharmonics seen in hypokinetic dysarthria $[36,42]$. In addition, the characteristics that differentiate connected speech from sustained phonation, such as rapid pitch and loudness variations, noise production during consonant production and the short voicing segments in connected speech [39], increase errors in the time-based measures yielding invalid results for connected speech, especially with severely dysphonic speakers [43].

In contrast to period-based measurement approaches, spectral/cepstral measures of dysphonia have been reported to be sensitive for quantifying the degree to which harmonics dominate the spectrum and therefore the strength of periodicity in the voice. These measures, moreover, do not rely on automated identification of cycle boundaries and are therefore not vulnerable to the limitations noted for traditional period-based techniques. In addition, cepstral measures have been validated for estimating dysphonia severity for a variety of phonation tasks [44-46]. Furthermore, the utility of these measures has been supported for differentiating typical and disordered voice production [47].

Spectral/cepstral analysis of dysphonia produces several useful acoustic voice parameters. CPP provides an indication of the relative amplitude of the dominant harmonic in the voice signal. CPP standard deviation (SD) provides an indication of the CPP variability over time. An indication of the dominance of spectral energy in the low frequency region, typically below $4 \mathrm{kHz}$, is provided by the $\mathrm{L} / \mathrm{H}$ SR as opposed to high frequency energy above $4 \mathrm{kHz}$. L/H SR SD provides an indication of the L/H SR variability over time [48]. Furthermore, these measures can be incorporated into specific combinations used in the prediction of perceived dysphonia severity (e.g., the Cepstral Spectral Index of Dysphonia (CSID). CSID values may be calculated via specific equations for sustained vowels, CAPE-V sentences, and for sentences taken from the Rainbow Passage [47]: Table 1 for descriptions of these measures.

In sustained phonation, a typical quasiperiodic signal is expected to exhibit well-defined Fo and harmonic structure associated with steadiness and consistency in its quality, pitch, and loudness over time, which is indicated by high amplitude CPP. In contrast, dysphonic voice is characterized by its decreased CPP amplitude and associated with an increase in its variability over time. In connected speech, typical speakers tend to have an increased CPP variability over time due to their vocal mechanism transitioning between voice/voiceless consonant and the normal variation of pitch and loudness. In contrast, in dysphonic voices CPP tends to exhibit decreased variability in connected speech [49]. In sustained phonation, normal speakers tend to have a high L/H SR due to the con-

Table 1. Measure Names and Descriptions

\begin{tabular}{ll}
\hline Measure names & \multicolumn{1}{c}{ Descriptions } \\
\hline Cepstral Peak Prominence (CPP) & dB value by which Cepstral Peak exceeds cepstral baseline \\
CPP Standard Deviation (CPP-SD) & Overall standard deviation of CPP measures \\
L/H Spectral Ratio (L/H SR) & $\begin{array}{c}\text { Spectral energy below 4 kHz to energy above } 4 \text { kHz expressed as a ratio. Normal voice signal tends to } \\
\text { have greater low frequency energy than high frequency energy (KayPENTAX, 2011) }\end{array}$ \\
L/H SR Standard Deviation (LH SR SD) & Overall standard deviation of L/H SR measures \\
Cepstral/Spectral Index of Dysphonia (CSID) & $\begin{array}{c}\text { A weighted sum of CPP, L/H SR, and L/H SR SD measures determined by regression analysis to fit } \\
\text { dysphonia ratings from the Rainbow Passage (Awan et al. 2009) }\end{array}$ \\
\hline
\end{tabular}


centration of the voice energy in the frequency region below 4 $\mathrm{kHz}$ while dysphonic voice tends to have a reduced L/H SR due to their high frequency energy above $4 \mathrm{kHz}$. In connected speech, normal speakers tend to have increased L/H SR variability over time due to their vocal mechanism transitioning between voiced/voiceless consonants and the variation of pitch and loudness across phrases and utterances. On the other hand, dysphonic voices tend to have a decreased $\mathrm{L} / \mathrm{H}$ Ratio variability in their connected speech productions [39].

Several authors have supported the use of spectral/cepstral measures to describe voice characteristics in connected speech [38,39,44,45,47,50-53]. While there have been several reports of spectral/cepstral analyses in the vowel productions of PD patients, few studies to date have looked at spectral/ cepstral measures in PD speech samples. Cepstral study of sustained phonation in untreated PD revealed reduced CPP in comparison to healthy participants [54]. In addition, low $\mathrm{CPP}$ and $\mathrm{L} / \mathrm{H}$ ratio, and higher CSID were demonstrated in untreated PD in comparison to healthy participants [55]. The acoustic spectral voice characteristics of PD include abnormally increased spectral slope, which correlates with listeners' judgments of dysarthria severity and is indicative of breathiness and incomplete glottal closure [36,56]. In treated PD, reductions in the harmonic spectral slope and decreased spectral tilt have been observed in two case studies of persons with PD after LSVT [28,57]. Furthermore, it was reported that CPP, $\mathrm{L} / \mathrm{H}$ ratio, and CSID in sustained phonation improved significantly in sustained vowels following LSVT, and the L/H SR energy redistributed into the higher frequency region of the spectrum [26]. To the best of the authors' knowledge, only one study has investigated spectral/cepstral characteristics of PD voice quality in the connected speech of untreated PD speakers. The study revealed a reduced CPP in comparison to healthy participants [55]. Investigating alternative spectral/cepstral measures such as the CSID in connected speech will clarify how well this composite measure is suited for speakers with PD. Thus, specifying the spectral/cepstral characteristics of a specific population such as PD should enhance our understanding of how connected speech may improve in this population following voice treatment.

The present research was not intended to evaluate the clinical effectiveness of LSVT, which for at least two decades has been well established in the literature. Instead, LSVT was used here as a clinically relevant approach to induce and habituate changes associated with increased overall voice intensity and closure of the vocal folds $[27,58]$ and thereby evaluate the effectiveness of spectral/cepstral measures. The primary goal was to study the effect of LSVT on cepstral/spectral analyses in connected speech for the purpose of understanding acoustic voice quality change for this important task in a group of speakers with PD. An additional goal was to examine descriptively the effect of task type for the PD group as a whole, comparing cepstral/spectral analyses findings in connected speech with findings previously observed in sustained phonation [26]. Finally, due to the heterogeneous nature of speakers with PD, it was also of interest to examine individual differences in spectral/cepstral measures obtained from the connected speech and sustained phonation tasks after LSVT. This will provide a better understanding of how phonation in the context of articulation and prosodic variations of connected speech may differ from the previously reported spectral/cepstral findings for sustained phonation within the same individuals.

\section{METHODS}

Participants were referred to the study by neurologists from the area in and around Memphis, Tennessee. Referral criteria included a firm diagnosis of $\geq 1$ year post-onset idiopathic $P D$, absence of other neurological disorders or history of speech or language disorders other than PD, presence of speech or voice difficulty, and no more than mild cognitive impairment or mild depression. The neurologists also provided motor scores from the Unified Parkinson's Disease Rating Scale (UPDRS) [59]. Additionally, an otolaryngologist performed a laryngeal endoscopic examination for each participant to rule out existing VF pathologies that may contraindicate participation in high-effort voice treatment. Prior to inclusion in the study, participants were evaluated by an experienced, ASHA certified speech-language pathologist (SLP) to verify the presence of hypokinetic dysarthria with hypophonia and overall dysarthria severity using the Mayo Clinic Rating Scales [5]. Twelve participants were initially enrolled in the study; however, two participants subsequently withdrew before completion because the strict treatment schedule could not be maintained. Another enrolled participant who completed the broader study was excluded from the present spectral/cepstral analysis due to severe vocal strain, as cepstral analysis has been found to be less effective in characterizing highly strained voices [44].

Nine speakers were included in the present analysis, six males and three females. Participants ranged in age from 52 
to 81 years $($ mean $=65, \mathrm{SD}=11.2)$, while years post diagnosis ranged from 2 to 27 years with a mean of 11.33 years. Individual speaker's dysarthria severity ranged from mild (rating of 1 ) to Severe (rating of 4) determined using the Mayo Clinic Rating Scales (Duffy, 2013). The present sample included two ratings of mild (ratings $=2$ ), four Moderate (ratings $=3$ ), two Marked (ratings $=3$ ) and two Severe (ratings $=4$ ) with a median of 2. Eight of the nine included participants were determined to have a substantial intelligibility impairment. Participant's percent intelligibility in single words ranged from $29 \%$ to $62 \%$ with a mean of 52 as measured by the Assessment of Intelligibility of Dysarthric Speech [60]. UPDRS Motor scores ranged from 7 to 49 with a mean of 24. See Alharbi et al. [26] for additional clinical description.

The enrolled participants continued to follow their regular schedule for anti-Parkinson medication prescribed by their physicians for the duration of the study and no additional speech or voice treatment was provided aside from LSVT. No participant had received LSVT prior to participating in the study. Other than having the same diagnosis and no prior speech or voice treatments, the sample of speakers with PD recruited for this study was clinically diverse. Variable characteristics of the sample included the following: one participant had a history of bilateral pallidotomy, three participants used hearing aids; one participant did not take anti-Parkinson medication; six participants were ambulatory, two participants used walkers with assistance and one participant was a wheelchair user. Each participant was administered LSVT in accordance with the standard protocol, consisting of 16 onehour sessions for four days per week across four consecutive weeks, by an ASHA certified SLP who was also certified in LSVT. Treatment sessions were scheduled from Monday to Thursday, leaving Friday for makeup sessions.

Speech recordings were obtained on three different days within one week before and one week after LSVT. Stimuli included reading the first paragraph of the rainbow passage [61] as part of a larger assessment protocol. Each connected speech signal was excerpted from the first paragraph of the Rainbow Passage with only the second and third sentences of the passage being analyzed in accordance with ADSV protocol. During the pre- and post-treatment assessments, no cues were given to the patients to produce loud speech. A head-mounted condenser microphone (AKG C420) with a flat frequency response below $10 \mathrm{kHz}$ was used. Recordings were made in a sound booth with the microphone $4 \mathrm{~cm}$ from the corner of the speaker's lips and out of the breath stream [62]. Using Kay-
Pentax CSL 4300B hardware (KayPENTAX, Montvale, NJ), signals were directly digitized to disc at a sampling rate of 44.1 kHz. KayPENTAX Analysis of Dysphonia in Speech and Voice (ADSV v.3.4.2) software (KayPENTAX, Montvale, NJ) was used to perform acoustic analyses. In order to approximate the 25 $\mathrm{kHz}$ standard for both ADSV analysis and CSID [39], signals were first down-sampled to $22.05 \mathrm{kHz}$. Connected speech file onset were trimmed to assure a $50 \mathrm{~ms}$ silent period before and after each production. Extraneous noise, such as silence, coughs, throat clears, et cetera, could impact the results. Therefore, such unwanted sections were trimmed and removed as needed from otherwise satisfactory acoustic signals.

Changes in connected speech intensity were calculated with reference to recorded calibration tones of known sound pressure level following procedures outlined by Buder and Cannito (2009). First, the voltage (root mean square, RMS) of the calibration tone was measured from the recording. Then RMS quantities obtained from the connected speech samples were standardized by the obtained values placed on a dB SPL scale relative to the calibration tone using the formula $20 \times$ $\log \left(\mathrm{RMS}_{\text {voice }} / \mathrm{RMS}_{\mathrm{Cal}}\right)$. Measures of interest were extracted from the output of the Analysis of Dysphonia in Speech and Voice (ADSV) program which included CPP, CPP-SD, the Low/High Spectral Ratio (L/H SR) measure with the default 4 $\mathrm{kHz}$ cutoff, the Low/High Spectral Ratio Standard Deviation (L/H SR-SD), and the CSID. The CSID value for the Rainbow Passage was computed using Excel from ADSV output using the following regression formula reported in Awan et al. (2009), which includes CPP, L/H SR, and L/H SR SD:

$$
\begin{aligned}
\mathrm{CSID}_{\mathrm{R}}= & 154.59-(10.39 \times \mathrm{CPP})-(1: 08 \times \mathrm{L} / \mathrm{H} \mathrm{SR})-(3.71 \times \mathrm{L} / \mathrm{H} \\
& \text { SR-SD }) .
\end{aligned}
$$

Intra- and inter-observer ADSV reliability measurements were calculated by trained SLP graduate students for $67 \%$ of files for sustained vowels and $51 \%$ of sound files for connected speech per participant productions. For these reliabilities, all connected speech files were segmented again for from longer recordings and were then re-trimmed, including for silent periods. A 4-month period passed before data were reanalyzed for intra-rater reliability. For ADSV measures, connected speech reliability was high for both inter-rater $(r>0.90$, $p<0.001)$ and intra-rater $(r>0.90, p<0.001)$ comparisons.

The present study included four stages of analysis. First, it was necessary to evaluate the effect of LSVT in terms of its primary target variable, acoustic voice intensity $\left(\mathrm{dB}_{\text {spl }}\right)$, on the connected speech production of current sample of speakers with PD. This was accomplished by comparing $\mathrm{dB}$ levels of 
their recorded Rainbow passages to test the assumption based on extensive prior literature that $\mathrm{dB}$ would increase significantly from pre-to-post treatment. Confirmation of this prediction was required to motivate further evaluation of the experimental hypothesis with respect to spectral/cepstral measures; a paired-samples t-test was computed at alpha level $=0.05$ (two-tailed).

The second stage of analysis was to examine the experimental hypotheses regarding the effect of LSVT on selected spectral/cepstral measures of voice in connected speech. A twoway repeated measures ANOVA was used to examine changes in spectral/cepstral measures for connected speech from preto-post LSVT. Within-participant variables were Treatment Period (Pre- $\mathrm{T}_{\mathrm{x}}$, Post $-\mathrm{T}_{\mathrm{x}}$ ) and Recordings Days (days 1, 2, and 3 Pre- $T_{\mathrm{x}}$ and days 1, 2, and 3 Post- $\mathrm{T}_{\mathrm{x}}$ ). An overall $\alpha$-level $=0.05$ was Bonferroni adjusted to 0.0125 for testing the significance of effects for each of the four acoustic variables to counteract the problem of multiple comparisons [63]. Effect sizes associated with the ANOVAs were calculated using classical eta squared against criteria of small $\geq 0.01$, medium $\geq 0.06$, large $\geq 0.14$ [64].

The third stage of analysis was to descriptively compare changes in connected speech with changes in sustained vowels reported in a previous study [26]. This was conducted by simply comparing which spectral/cepstral variables that improved significantly from pre-to-post treatment for each speaking condition and also by inspecting the means and SDs from both studies. The sustained vowel data was taken from Alharbi et al. [26] and is used here with permission of the journal editor. The fourth stage of analysis was to examine individual differences in both connected speech and sustained phonation in response to LSVT. A change criterion of one-half SD of the post treatment distribution was employed; this value was adopted from the standard criterion for identifying a meaningful change using the Cohen's $d$ statistic [65]. Because one-half SD is generally regarded as a moderate effect size it provides a benchmark for a meaningful pre- to post- treatment increase or decrease in individual participant's vocal function. Each participant's improvement was calculated relative to the differences between the pre and post treatment values for both sustained vowel and connected speech. For each participant the four acoustic variables were averaged pre-treatment and post-treatment across assessment days for connected speech. In the previous study, sustained vowel data had been averaged across days and repeated trials within days.

\section{RESULTS}

In the current study, the first stage of analysis was to examine intensity changes to provide a validation of the treatment. Results indicate that before LSVT, the speakers' mean intensity across days of connected speech was $78.8 \mathrm{~dB}_{\text {spl }}$ (note that this value reflects a $4 \mathrm{~cm}$ microphone to mouth distance, so it is not comparable with lower values regarded to be typical of conversational speech at typical speaker-to-speaker distances or with those reported by researchers recording with more distant microphone placements, e.g. $30 \mathrm{~cm}$ ). Following treatment, the mean connected speech intensity was $81.7 \mathrm{~dB}_{\text {spl }}$. This increase in intensity for the connected speech task preto-post treatment was statistically significant (paired samples $t(8)=2.32, p=0.049)$, which is in keeping with expectations of the treatment program.

The second stage of analysis addressed the primary goal for the current study, which was to examine the effect of LSVT on selected spectral/cepstral measures of voice in connected speech for participants with PD. Table 2 provides the means and SDs for each measure obtained from the PD participants' connected speech before and after LSVT, averaged across recording days. A statistically significant difference from pre-topost LSVT was found for one acoustic variable: $\operatorname{CPP}(F(1,8)=$ 10.51, $p=0.012, \eta^{2}=.08$ ). The other acoustic variables did not differ significantly from pre-to-post treatment at the Bonfer-

Table 2. Connected speech and sustained phonation means and (SD)s of measures from patients with PD before and after treatment

\begin{tabular}{lccccc}
\hline \multirow{2}{*}{ DV } & \multicolumn{2}{c}{ Connected Speech* } & & \multicolumn{2}{c}{ Sustained Phonation** } \\
\cline { 2 - 3 } \cline { 5 - 6 } CPP & Pre & $5.96(1.23)$ & $6.67(1.25)$ & Pre & $12.35(1.38)$ \\
CPP SD & $3.34(.49)$ & $3.63(.71)$ & & $1.60(1.72)$ & $1.25(.33)$ \\
L/H SR & $35.30(4.06)$ & $36.12(5.24)$ & $36.47(5.50)$ & $34.60(5.60)$ \\
CSID & $13.83(16.59)$ & $6.47(15.66)$ & & $35.84(13.80)$ & $21.87(10.18)$ \\
\hline
\end{tabular}

${ }^{*}$ See text for review of statistical significance for these treatment changes.

**The sustained phonation data was originally published in Alharbi et al. (2019) and is used here with permission. 
roni-corrected alpha levels: CPP-SD $(F(1,8)=4.67, p=0.063$, $\left.\eta^{2}=.06\right), \mathrm{L} / \mathrm{H}$ SR $\left(F(1,8)=.64, p=0.445, \eta^{2}=.01\right)$, and CSID $\left(F(1,8)=9.56, p=0.015, \eta^{2}=.05\right)$. There were no statistically significant main effects or interactions for days.

Sample analyses are presented in Figure 1-these depictions reinforce the association between cepstral peak prominence and clearer harmonic structure throughout the spectrum, as well as the stronger energy at higher harmonics accounting for the possibility that a decrease in LH spectral ratio may be seen in a healthier voice relative to hypophonia; this observation also accords with spectrographic inspections of the voice recordings. It should be noted that CSID, while not strictly significant at the family-wise adjusted alpha level, was very close to this cutoff and exhibited a strong effect size. Thus, the Holm-Bonferroni correction procedure was applied to counteract the problem of loss of power in multiple comparisons. After applying the Holm-Bonferroni correction procedure CSID exhibited a statistically significant effect ( $p=0.015$, given Holm-Bonferroni criterion of $p=0.016$ ) [63]. Though nonsignificant, the CPP-SD also showed a medium effect size and indicates that acoustic variables that are closely related to the CPP may be reasonable indices of treatment change.

The third stage of analysis was to investigate task-related differences for the group of speakers with PD both for connected speech and sustained phonation. Table 2 provides the means and SDs for each measure obtained from the PD participants' connected speech as well as for sustained vowels re-

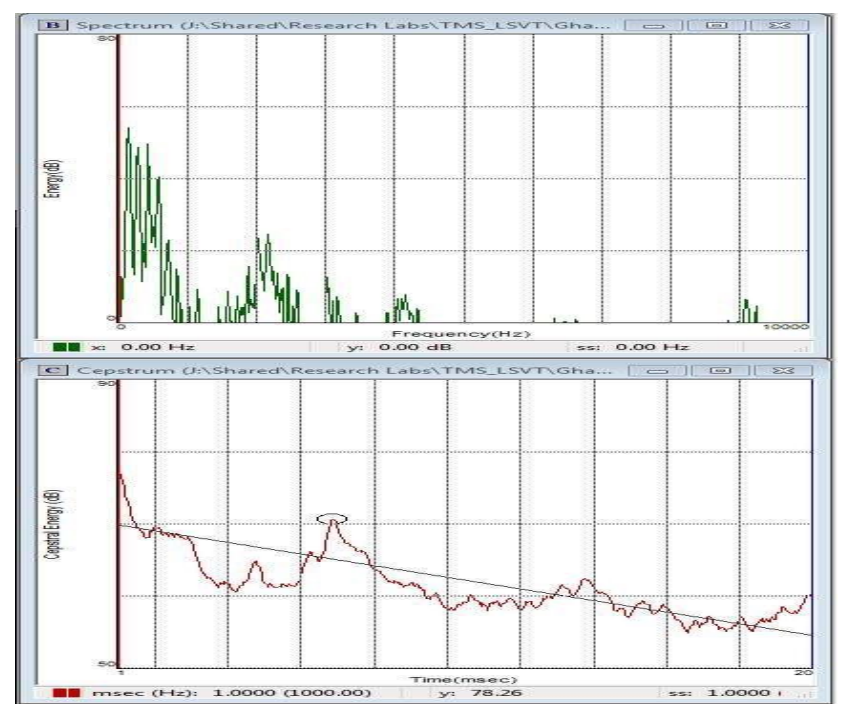

ported by Alharbi et al. [26], before and after LSVT, averaged across recording days and trials. The sustained vowel data is provided to compare pre-to-posttreatment change across the two speech elicitation tasks. In connected speech, CPP and CSID were statistically significant, while in sustained phonation three of the acoustic variables were statistically significant including CPP, CPP-SD, and CSID.

The fourth stage of analysis was to examine individual differences in response to LSVT across the experimental measures and tasks. Table 3 summarizes the results for all participants, indicating those who met and who did not meet the criterion (0.5 SD) for the selected ADSV measures for both connected speech and sustained phonation, and which variables improved for each participant. Intensity improvement following LSVT on sustained vowels was demonstrated by seven speakers versus six speakers who showed speech intensity improvement in connected speech (Figure 2 for individual treatment effect plots). CPP attained the 0.5 standard deviation criterion for seven participants in sustained phonation vs. five participants who attained this criterion in connected speech (Figure 3), with four participants who showed an improved CPP in both sustained phonation and connected speech, and one participant who showed improved CPP in connected speech but no improvement of CPP in sustained phonation. All participants with PD demonstrated a reduction in their CPP variability (CPP-SD) in sustained phonation, while four speakers demonstrated an increased CPP-SD fol-

Figure 1. Rainbow Passage production of PD participant \#5 demonstrating increased harmonic structure and reduced spectral noise pre- to post-treatment (Top panels). Bottom panels demonstrate increased CPP from pre- to post-treatment. Pre-treatment CPP $=5.34$ and CSID $=26.41$. Post-treatment CPP $=7.46$ and $\mathrm{CSID}=1.01$.

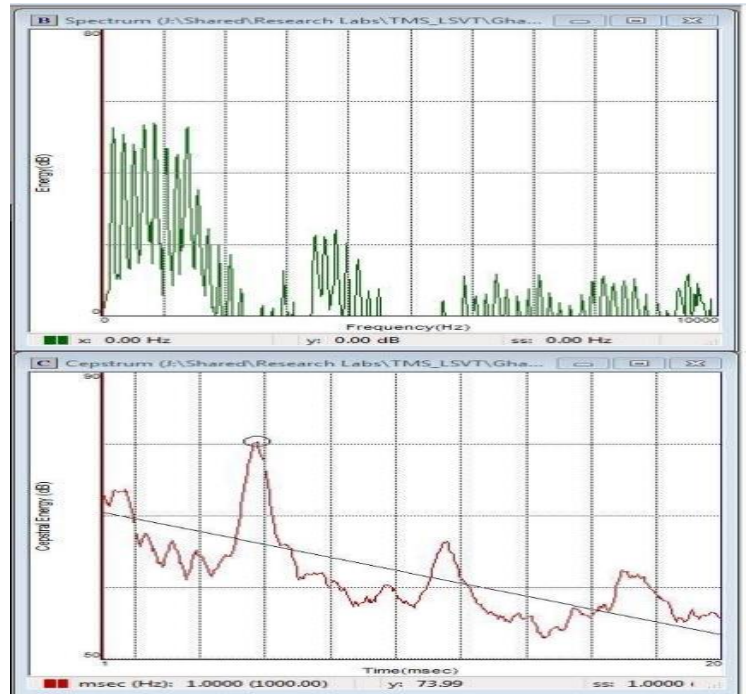


Table 3. Individual differences in response to treatment for ADSV selected measures for both sustained phonation and connected speech

\begin{tabular}{|c|c|c|c|c|c|c|c|c|c|c|}
\hline \multirow{2}{*}{$\begin{array}{l}\text { Participant } \\
\text { Measure: }\end{array}$} & \multicolumn{5}{|c|}{ Sustained phonation } & \multicolumn{5}{|c|}{ Connected speech } \\
\hline & $\mathrm{dB} S P L$ & CPP & CPP-SD & L/H SR & CSID & $\mathrm{dB} S P L$ & CPP & CPP-SD & L/H SR & CSID \\
\hline Post-TX .5 SD: & $>2.89$ & $>.62$ & $<.11$ & $<.62$ & $<4.11$ & $>2.45$ & $>.62$ & $>.38$ & $>2.61$ & $<4.11$ \\
\hline 4 & * & * & * & * & * & * & * & * & & * \\
\hline 5 & * & * & * & * & * & * & * & * & & * \\
\hline 6 & * & * & * & & * & * & $*$ & * & * & * \\
\hline 1 & * & * & * & * & * & & * & * & & * \\
\hline 8 & * & * & * & * & * & * & & & & \\
\hline 9 & * & & * & & * & $*$ & & & * & \\
\hline 3 & * & * & * & & * & * & & & & \\
\hline 2 & & * & * & & & & & & * & \\
\hline 10 & & & * & * & & & * & & & \\
\hline
\end{tabular}

Asterisks indicate which measures met the 0.5 SD Post vs Pre criterion.
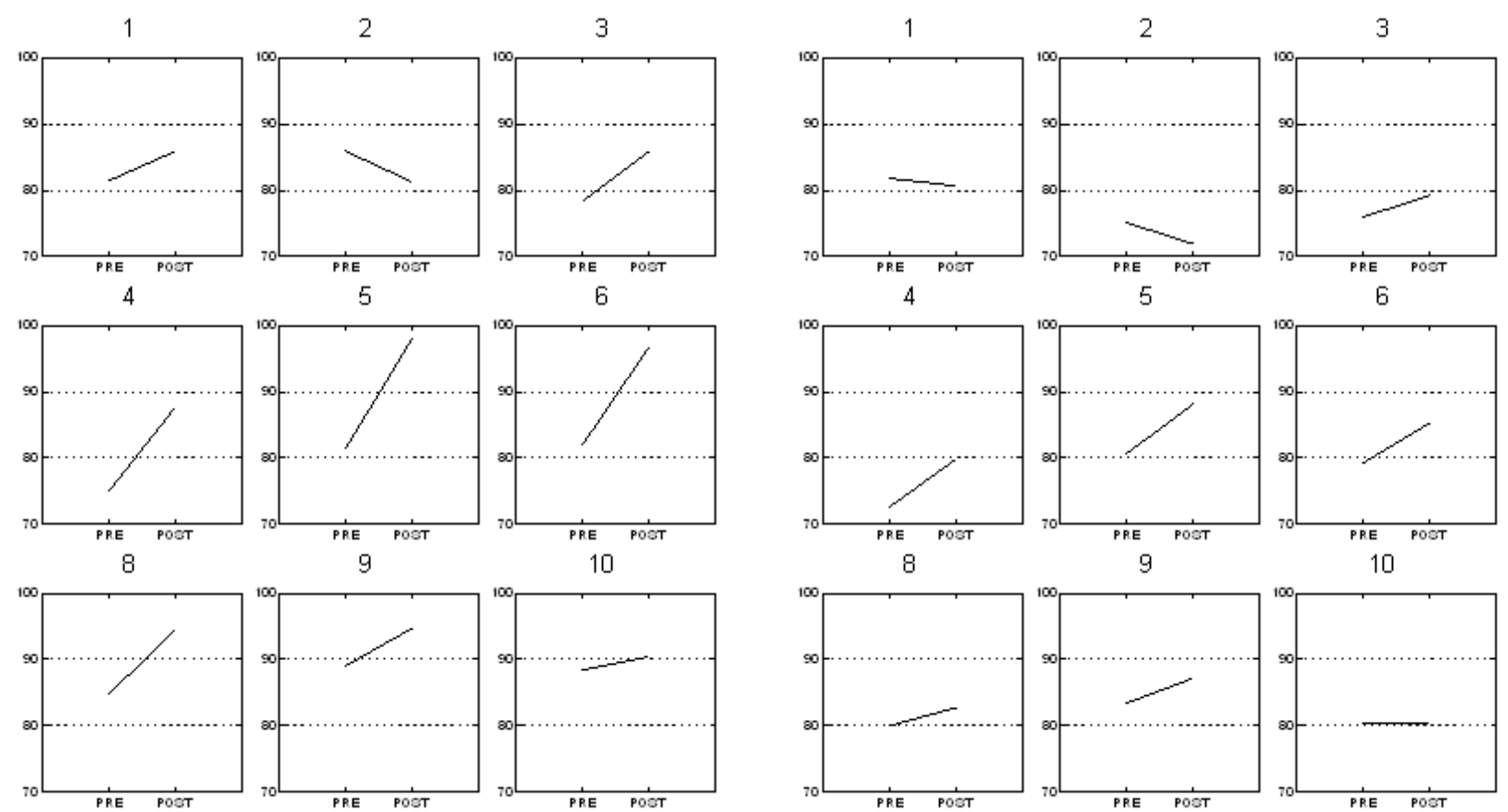

Figure 2. Line graphs of average pre-Tx and post-Tx values for vocal intensity changes per participant for sustained phonation (left panels) and connected speech (right panels).

lowing LSVT in connected speech (Figure 4). In the measure of L/H SR in sustained phonation, five speakers showed a reduction in L/H SR. For connected speech, three speakers demonstrated an increase in L/H SR following treatment, which suggests an increase in low frequency energy following treatment (Figure 5). An overall reduction of dysphonia severity as summarized by CSID on sustained phonation was observed in eight speakers, while in connected speech an overall dysphonia severity reduction was demonstrated in four speakers (Figure 6).

\section{DISCUSSION}

The primary purpose of this study was to assess the utility of selected spectral/cepstral measures for demonstrating acoustic voice changes in the connected speech of individuals with PD in connected speech following LSVT. Spectral/cepstral analyses also were used to descriptively compare changes in 

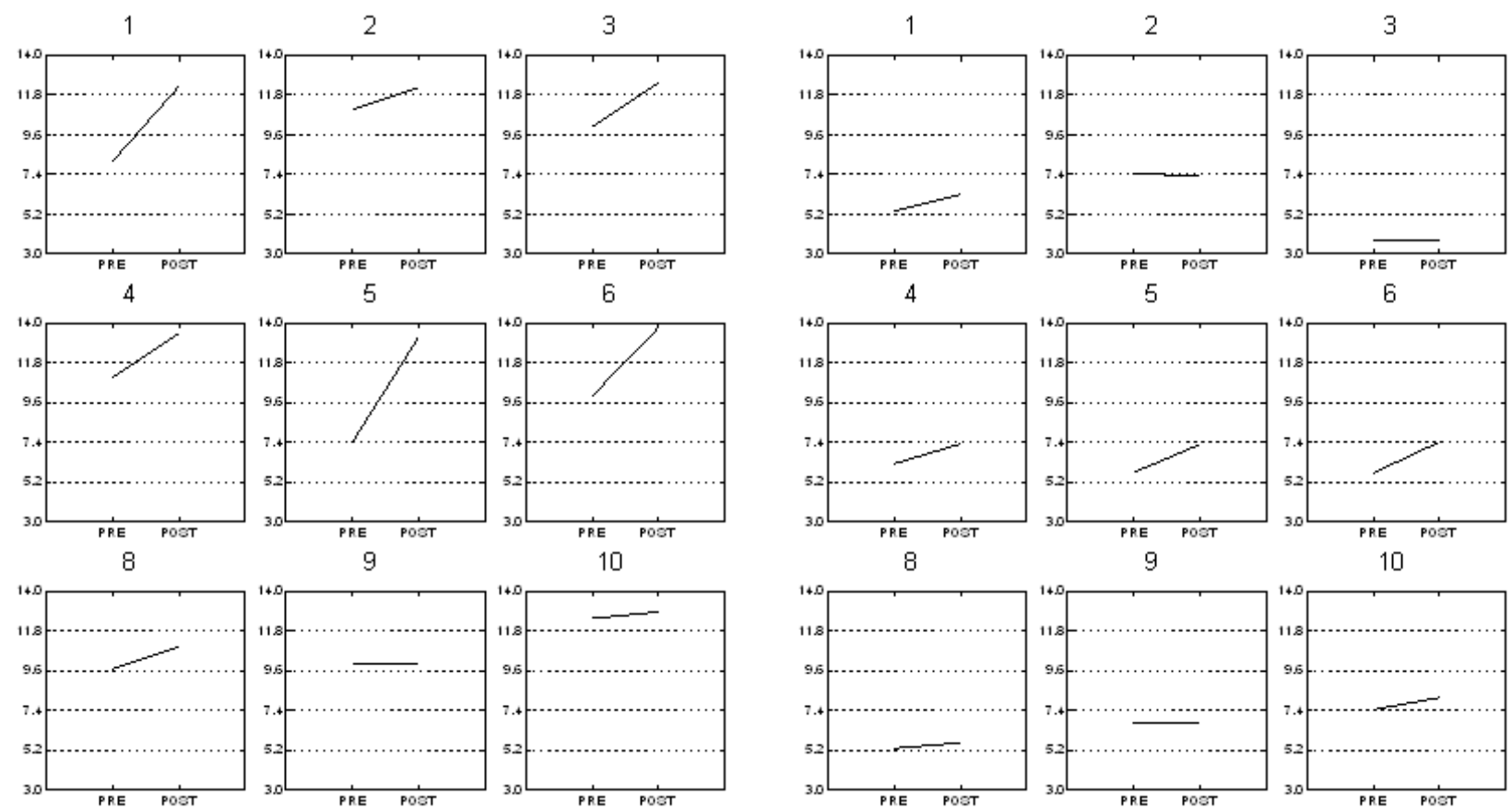

Figure 3. Line graphs of average pre-Tx and post-Tx values for CPP per participant for sustained phonation (left panels and connected speech (right panels)
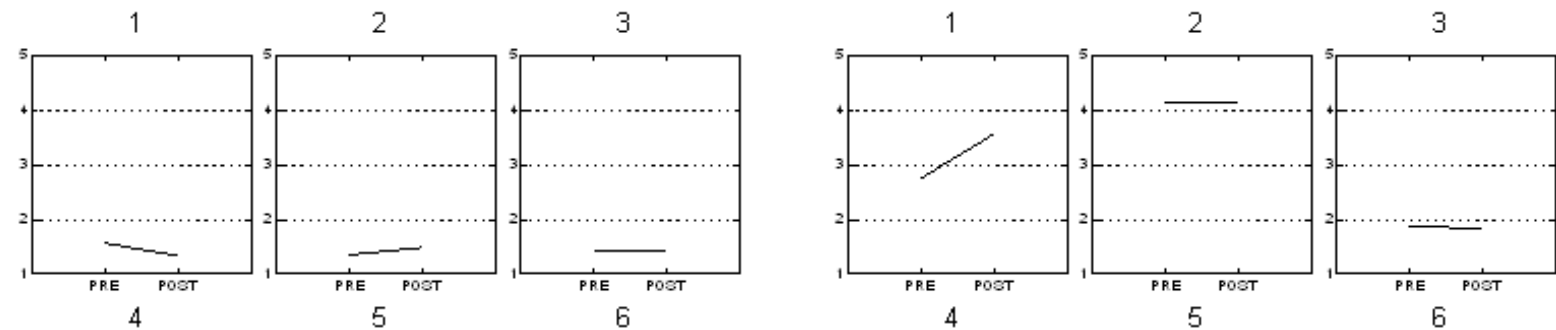

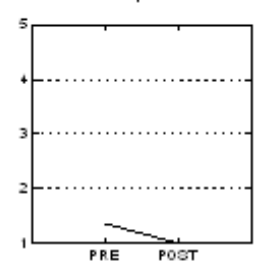

8
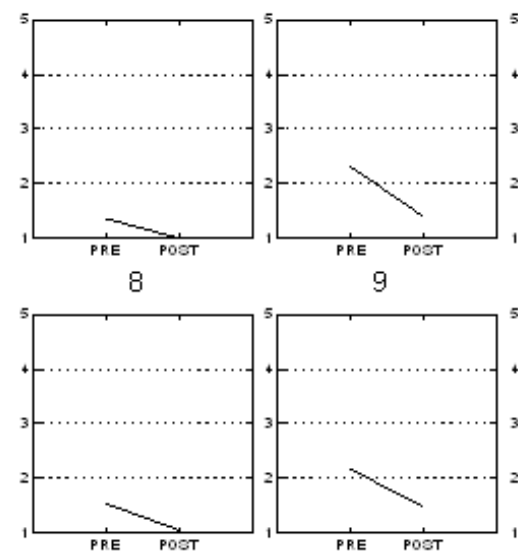

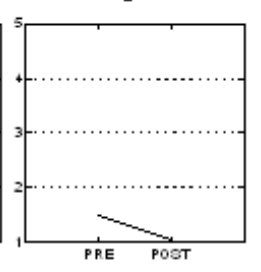

10

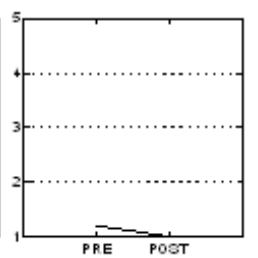

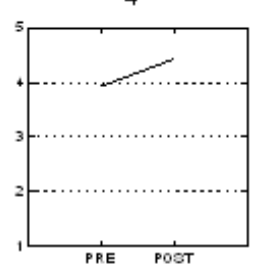

8

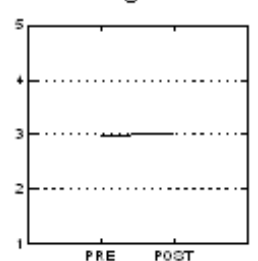

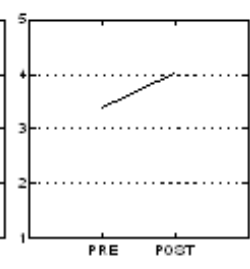

10
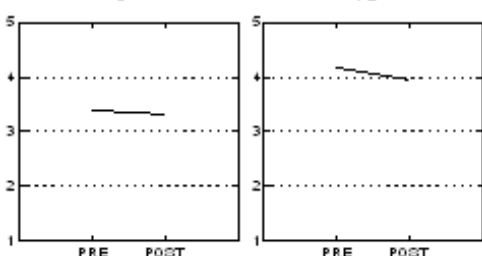

PRE POST

Figure 4. Line graphs of average pre-Tx and post-Tx values for CPP-SD for sustained phonation (left panels) and connected speech (right panels).

connected speech with changes in sustained vowels reported in a sustained phonation study as reviewed in [26]. An additional goal was to examine individual differences in responses to LSVT across the experimental measures.

\section{Effect of LSVT on spectral/cepstral measures in connected speech and sustained phonation}

The spectral/cepstral analyses demonstrated that out of the four spectral/cepstral variables chosen, CPP and CSID were 


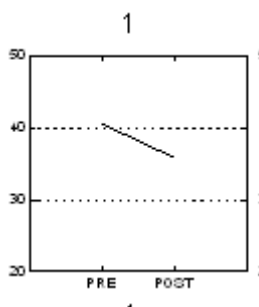

4

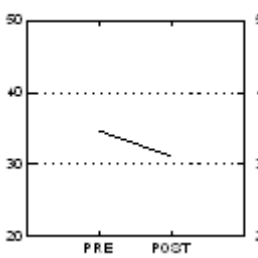

8

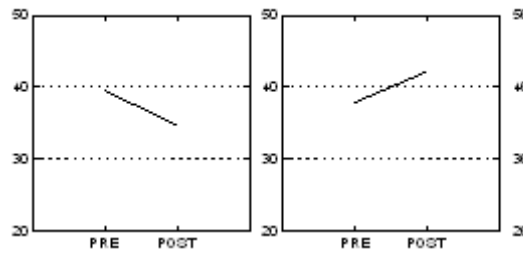

3
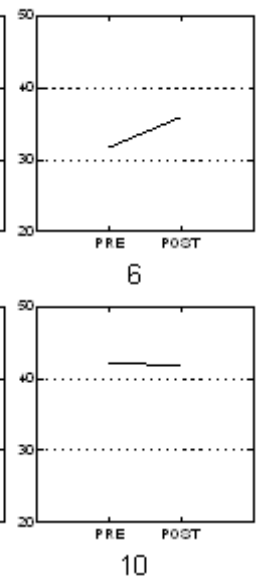

10

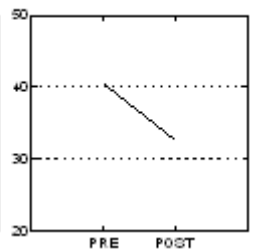

2

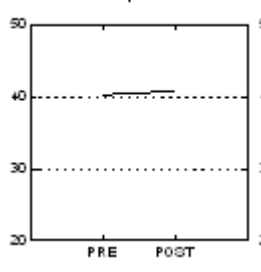

4
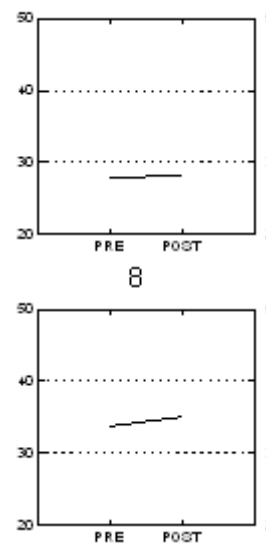

3
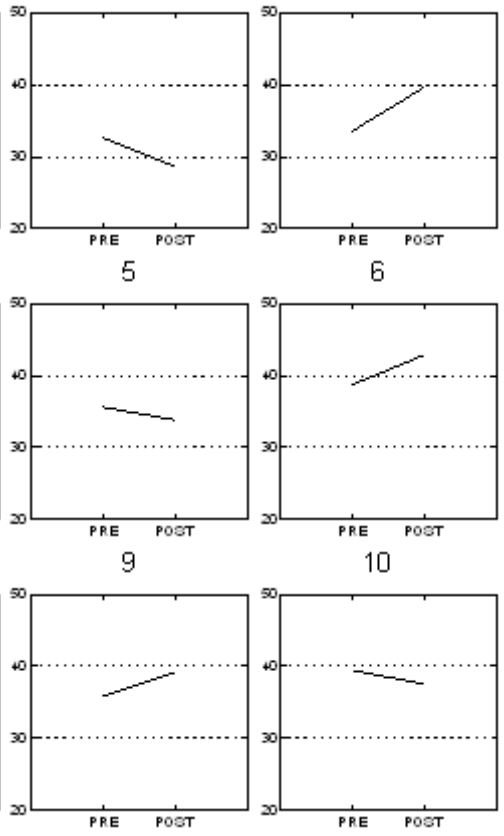

Figure 5. Line graphs of average pre-Tx and post-Tx values for L/H SR for sustained phonation (left panels) and connected speech (right panels).

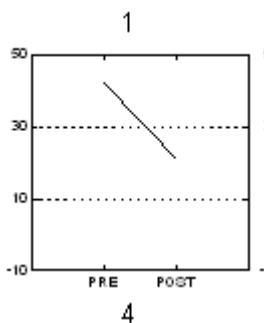

2

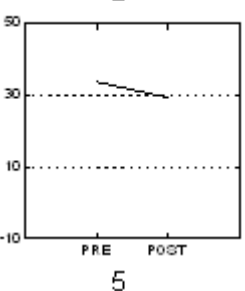

5

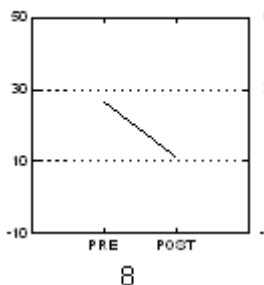

8

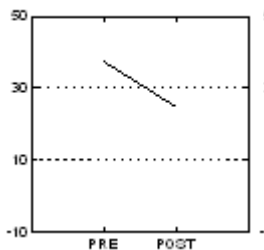

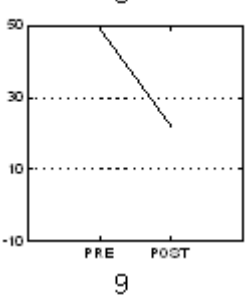

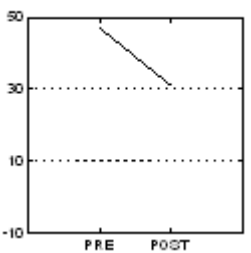

3
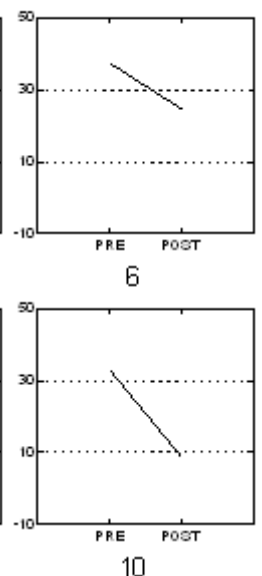

10

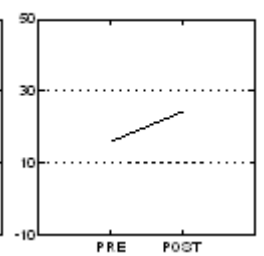

1

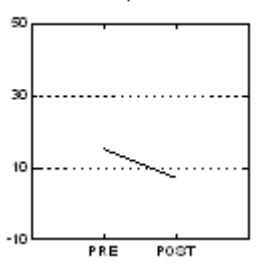

4

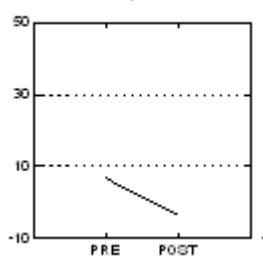

8

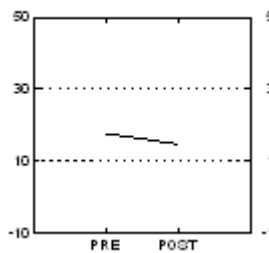

2

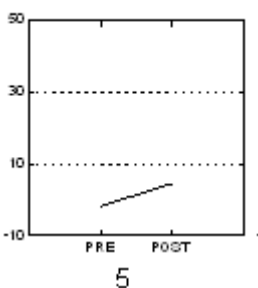

5
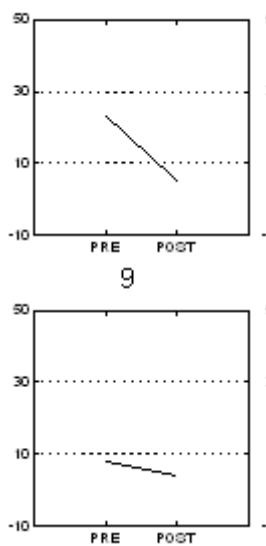

3

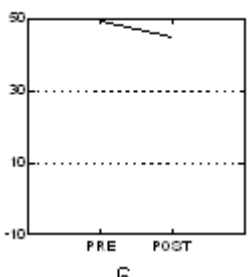

6

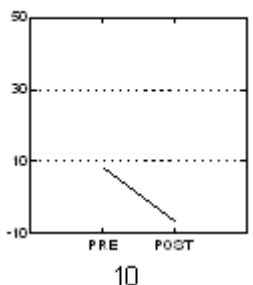

10

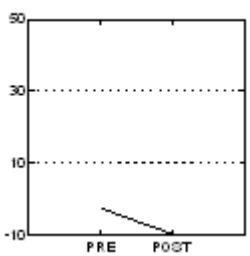

Figure 6. Line graphs of average pre-Tx and post-Tx values for CSID for sustained phonation (left panels) and connected speech (right panels).

the only measures that detected statistically significant treatment related changes in connected speech. The finding that CPP was most sensitive to spectral changes resulting from LSVT is consistent with improved harmonic structure across the voice spectrum; spectrographic inspection of our samples affirmed that harmonics were not just clearer in low frequencies but extended into higher frequency ranges post-treatment, as illustrated in Figure 1. Even with the occurrence of 
laryngeal and supra-laryngeal articulation and prosodic variations characteristic of connected speech, CPP was shown to be the most sensitive cepstral measure to LSVT in that it was not influenced by speaking condition, which is in agreement with Watts \& Awan (2011). However, while it is true that CPP improved for both speaking conditions, the current study demonstrated that the magnitude of the CPP was greater for sustained vowels than for connected speech.

The spectral changes observed in this study primarily reflected in the CPP indirectly support previous studies which have found that following intensive voice therapy there is increased glottal closure [30], increased subglottal pressure, and decreased EGG open quotient post LSVT [28,31]. While previous studies have indicated that CPP may be sensitive to vocal loudness/intensity variations [66,67], the correlation between pre-post change in CPP and intensity accounted for a trivial amount of variance $\left(r^{2}=.02\right)$ in the current study. In addition, as seen in Table 3 in connected speech participant \#1 and in sustained phonation speech participant \#2 improved their CPP but not their vocal intensity. Together, these observations demonstrate that some participants may improve their harmonic structure without increasing vocal intensity. This finding is also supported by the moderate correlation between $\mathrm{CPP}$ and $\mathrm{dB}$ treatment changes that had been found in sustained phonations [26].

Turning to the composite index, the fact that CSID was also statistically significant in both sustained phonation and connected speech tasks reinforces prior findings that overall voice quality improved following LSVT treatment. This finding also supports the clinical utility of spectral/cepstral measures for documenting treatment-related voice change in PD post LSVT $[26,43]$. The present acoustic finding of decreased dysphonia is also consistent with previous findings of improved perceptual voice quality after LSVT in connected speech [11]. Taken together with the findings of Alharbi et al. [26], this study demonstrated that CSID is a measure that can detect treatment effects for this population in both connected speech and sustained phonation. Absence of statistically significant main or interaction effect involving the factor of days (i.e., daily recording sessions) indicates the stability of participants' phonatory function prior to and following LSVT. Therefore, improvements demonstrated from pre-to-post treatment in this study cannot be attributed to day-to-day variability or to practice effects.

CPP-SD in the connected speech condition failed to demonstrate any significant changes following treatment. How- ever, the present findings that CPP SD showed an increase with treatment in connected speech with a medium effect size is informative, given Alharbi et al.'s [26] finding that CPP SD was also an explanatory treatment outcome measure when analyzing sustained phonation; Watts \& Awan (2011) had also demonstrated that CPP-SD may be an effective measure in examining treatment outcome on speakers with dysphonia. The small sample size or the number of dependent variables $(M=4)$ may have affected the power for statistics to reveal a significant difference in the current study; this may also be related to the large range of severity in the present group of speakers with PD. The observation of a medium effect size for treatment nonetheless suggests that this variable may prove to be a significant marker for voice improvement in connected speech with a larger sample of speakers with PD.

L/H SR did not show any significant change in response to treatment in connected speech. This finding was not consistent with findings reported by Watts \& Awan (2011), who found that $\mathrm{L} / \mathrm{H}$ SR was able to demonstrate treatment related change in individuals with dysphonia of various etiologies. In addition, the finding was not consistent with Gillespie et al. (2014) who demonstrated that, in speech, the $\mathrm{L} / \mathrm{H}$ ratio and its SD may be an important outcome measure to track responses to behavioral intervention in single voice disorder, which included vocal fold lesions, primary muscle tension dysphonia, vocal fold atrophy or unilateral vocal fold paralysis before and after treatment. However, these discrepancies with Watts \& Awan (2011) and Gillespie et al. [51] may be because the previous studies did not examine participants with PD; the participants in this study may have had different degrees of breathiness or roughness than the participants in those previous studies. Additionally, the lack of significance of the $\mathrm{L} / \mathrm{H}$ SR on connected speech may be due to the default cut off frequency of $4 \mathrm{kHz}$ used in ADSV for computation of this spectral ratio. Previous findings for sustained phonation in speakers with PD suggested that restricting the L/H SR cutoff to a region just above the second formant and below the third formant increased the sensitivity of this measure to treatment effects in speakers with PD [26]. In connected speech, comparable adjustment of the L/H SR cutoff was not possible due to the variability of formant frequencies across the variety of vowels in the Rainbow Passage.

\section{Individual differences before and after LSVT on spectral/ cepstral measures}

CPP findings indicated that in the present sample, not all par- 
ticipants achieved the $.5 \mathrm{SD}$ criterion though as a group they still had better harmonic structure following treatment. The finding that fewer speakers improved on CPP in connected speech than in sustained vowels is not surprising: Watts \& Awan (2011), when evaluating the diagnostic value of spectral/cepstral measures to differentiate dysphonic speakers from healthy speakers, demonstrated a smaller effect of CPP in connected speech when compared to sustained phonation. In addition, when comparing the correlation of $\mathrm{CPP}$ with the dysphonia severity rating, a stronger correlation was observed in sustained phonation compared to connected speech [39]. The present findings agree with Watts \& Awan (2011) that, even within the same speaker, the degree of dysphonia severity and/or voice quality type may be more evident in sustained vowels than in connected speech.

As previously indicated the CPP-SD findings, though nonsignificant, yielded an effect size indicated a positive effect of LSVT for increasing some of the participants' speech variability in connected speech. CPP-SD had been the only measure which met the criterion for all participants in sustained phonation. It should be noted that the interpretation of the CPP SD will differ in speech vs. vowel contents. In the current study on connected speech, the increase in CPP SD may be consistent with an increased range of vocal frequency and intensity capability necessary for effective prosodic control vs. reduced CPP SD in sustain phonation post-treatment indicative of consistency in harmonic structure (Table 2).

L/H SR measures were not affected significantly in connected speech as a result of treatment; Alharbi et al.'s [26] finding of a decreased L/H SR in sustained phonations was clearly not found in connected speech where an overall slight increase was observed. In prior work on disordered voices other than PD, improved voice quality had tended to show an increase in the $\mathrm{L} / \mathrm{H} \mathrm{SR}$, consistent with the reduction of high frequency noise often associated with breathiness [39]. However, it is possible that in some cases, an increase in high frequency energy post-treatment and a decrease in the overall L/ H SR may also reflect positive change and may be due to (a) improved precision of consonant production and/or (b) an increase in the amplitude of high frequency harmonics. More studies are needed to understand the direction of change in the L/H SR in sustained phonation and connected speech materials following treatment in speakers with PD in relation to healthy controls.

Examining CSID findings in Table 3, it is notable that while many participants exhibited an overall improvement in this index for sustained phonation, a smaller number (\#s 1, 4, 5, 6) showed a reduction in CSID for connected speech. This finding suggests that some patients may respond to therapy differently in connected speech performance than in sustained phonation. Individual patients' conditions and severity (such as breathiness) may also influence which task is most responsive to therapy according to these measures. The differences between vowel versus speech tasks observed here reinforces the need for both sample types to be included in clinical assessments.

In summary, cepstral/spectral measures have been shown to be valid measures for estimating dysphonia severity in both sustained vowels and continuous speech $[45,46]$ and for differentiating typical and disordered voice production. The present study extends the use of these measures to speakers with PD for characterizing speech and voice aspects prior to treatment and for quantifying treatment outcomes. The present findings also support the use of LSVT as a treatment approach for improving voice quality in addition to intensity in PD. The current study supports the use of the CPP and CSID as outcome measures for documenting phonatory changes in connected speech following LSVT.

Inspection of individual differences demonstrated that participants \# 1, 4, 5, and 6 did improve on CPP, CPP-SD and CSID in both tasks, with more participants demonstrating an improvement in sustained phonation than in connected speech. In general, spectral/cepstral measures represented the degree of severity in voice quality in some of the participants. For example, a few participants did not show a vocal intensity improvement but did show slight spectral/cepstral measures improvement. The participants that exhibited this pattern were very mild breathy prior to treatment, as with participant \#2. In addition, participants who did show improvements were those who had a more breathy voice quality prior to treatment while following treatment there was no breathiness detected as seen in participants \#s 1 \& 5 . Present results agree with those obtained by Gillespie et al. [51] indicating that the benefits of spectral/cepstral measures such as CPP, CPP-SD, L/H SR, and CSID may vary depending upon the type of disorder being investigated. In addition, the present findings suggest that not all individuals will exhibit withinparticipant effects or the effects may be very small, and these may vary with different elicitation tasks.

\section{Limitations and Directions for Future Research}

In evaluating the outcomes of the current study, it is impor- 
tant to bear in mind that the present sample was both small in size and heterogenous in severity. Further studies are needed to replicate these findings with a larger sample size and to examine the potential effect of subject variability on the effectiveness of spectral/cepstral measures as treatment outcomes. Furthermore, it is important to extend ADSV analyses to phonation elicited at long-term follow up post LSVT. Future studies may also directly compare present spectral/cepstral measures with other harmonic spectral analytic techniques in both sustained vowels and connected speech produced by individuals with $\mathrm{PD}$, as well as comparison to physiological measures such as electroglottography (EGG) and video-laryngoscopy to document the underlying bases of spectral/cepstral improvement following LSVT. The relationship between spectral/cepstral measures and perceptual scaling judgements made by trained clinicians pre- vs. post-LSVT would also provide further insight into the interpretation of these acoustic measures.

\section{Statement of ethics}

Each participant signed a consent form approved by the Internal Review Board of the University of Memphis (Memphis, $\mathrm{TN}$ ), which is regulated by the US Department of Health and Human Services.

\section{CONFLICT OF INTEREST}

Dr. Ghadah G. Alharbi is employed at the University of Jeddah. She has no financial or nonfinancial relationships to disclose.

Dr. Michael P. Cannito is employed at University of Louisiana at Lafayette where he holds the Hawthorne/BORSF Professorship in Communicative Disorders. He has no nonfinancial relationships to disclose.

Dr. Eugene H. Buder is employed at the University of Memphis as an Associate Professor. He serves as a co-investigator on two NIH R01 grants (awarded to U. of Memphis Professor D. Kimbrough Oller, PI on the topic of infant vocal development) and one IARPA contract (awarded to U. of Memphis Professor Santosh Kumar, PI on the topic of workplace performance indicators); neither of these has any financial bearing on the current submission. He has no nonfinancial relationships to disclose.

Dr. S.N. Awan is Professor Emeritus from Bloomsburg University of PA. He is also a consultant to Pentax Medical/KayPentax (Montvale, NJ) for the development of commercial computer software including cepstral analysis of continuous speech algorithms. KayPentax licenses the algorithms that form the basis of the Analysis of Dysphonia in Speech and Voice (ADSV) program from Dr. Awan.

\section{FUNDING SOURCES}

This project was funded in part by the Ministry of Higher Education, Kingdom of Saudi Arabia.

\section{ACKNOWLEDGMENTS}

We gratefully acknowledge the contributions of Debra Suiter, Ph.D. and Teresa Wolf, M.S. in providing LSVT and participating in the pre-post evaluations.

\section{REFERENCES}

1. Parkinson's Disease Foundation. Statistics on Parkinson's New York: Parkinson's Disease Foundation. 2017; Retrieved from: http://www.PDF.org.

2. Fahn S, Bressman S, Marsden C. Classification of dystonia. Advances in neurology. 1998;78:1-10.

3. Miller N. Communication changes in Parkinson's disease. Practical Neurology. 2017;17:266-274.

4. Darley FL, Aronson AE, Brown JR. Speech disorders; Nervous system; Motor neurons; Diseases; Physiopathology. Philadelphia: Saunders; 1975.

5. Duffy JR. Motor speech disorders: Substrates, differential diagnosis, and management. 3rd ed. St. Louis, MO: Elsevier; 2013.

6. Ludlow CL, Bassich CJ, McNeil M, Rosenbek J, Aronson A. Relationships between perceptual ratings and acoustic measures of hypokinetic speech. The dysarthrias: Physiology, acoustics, perception, management. 1984:163-195.

7. Ramig L, Fox C, Sapir S. Speech disorders in Parkinson's disease and the effects of pharmacological, surgical and speech treatment with emphasis on Lee Silverman voice treatment (LSVT ${ }^{\circledR}$ ). Handbook of Clinical Neurology. 2007;83:385-399.

8. Hanson DG, Gerratt BR, Ward PH. Cinegraphic observations of laryngeal function in Parkinson's disease. The Laryngoscope. 1984;94:348-353.

9. Lam J, Tjaden K. Clear Speech Variants: An Acoustic Study in Parkinson's Disease. Journal of Speech, Language, and Hearing Research. 2016;59:631-646.

10. Tjaden K, Wilding GE. Rate and loudness manipulations in dysarthria: Acoustic and perceptual findings. Journal of Speech, Language, and Hearing Research. 2004;47:766-783.

11. Baumgartner CA, Sapir S, Ramig LO. Voice quality changes following phonatory-respiratory effort treatment $\left(\right.$ LSVT $\left.^{\circledR}\right)$ versus respiratory effort treatment for individuals with Parkinson disease. 
journal of Voice. 2001;15:105-114.

12. Ferrand CT. Speech science: An integrated approach to theory and clinical practice. Ear and Hearing. 2001;22:549.

13. Goberman AM, Coelho C. Acoustic analysis of Parkinsonian speech I: Speech characteristics and L-Dopa therapy. NeuroRehabilitation. 2002;17:237-246.

14. Flint AJ, Black SE, Campbell-Taylor I, Gailey GF, Levinton C. Acoustic analysis in the differentiation of Parkinson's disease and major depression. Journal of Psycholinguistic Research. 1992;21: 383-399.

15. Kent RD, Weismer G, Kent JF, Vorperian HK, Duffy JR. Acoustic studies of dysarthric speech: Methods, progress, and potential. Journal of Communication Disorders. 1999;32:141-186.

16. Kim Y, Kent RD, Weismer G. An acoustic study of the relationships among neurologic disease, dysarthria type, and severity of dysarthria. Journal of Speech, Language, and Hearing Research. 2011; 54:417-429.

17. Pinto S, Ozsancak C, Tripoliti E, Thobois S, Limousin-Dowsey P, Auzou P. Treatments for dysarthria in Parkinson's disease. The Lancet Neurology. 2004;3:547-556.

18. Rusz J, Tykalová T, Klempiŕ J, Čmejla R, Růžička E. Effects of dopaminergic replacement therapy on motor speech disorders in Parkinson's disease: longitudinal follow-up study on previously untreated patients. Journal of Neural Transmission. 2016;123:379387.

19. Halpern AE, Ramig LO, Matos CEC, Petska-Cable JA, Spielman JL, Pogoda JM, et al. Innovative technology for the assisted delivery of intensive voice treatment (LSVT ${ }^{\circledR}$ LOUD) for Parkinson disease. American Journal of Speech-Language Pathology. 2012;21:354367.

20. Sapir S, Spielman JL, Ramig LO, Story BH, Fox C. Effects of intensive voice treatment (the Lee Silverman Voice Treatment [LSVT]) on vowel articulation in dysarthric individuals with idiopathic Parkinson disease: acoustic and perceptual findings. Journal of Speech, Language, and Hearing Research. 2007;50:899-912.

21. Ramig L, Sapir S, Countryman S, Pawlas A, O'brien C, Hoehn M, et al. Intensive voice treatment $\left(\mathrm{LSVT}^{\circledR}\right.$ ) for patients with Parkinson9s disease: a 2 year follow up. Journal of Neurology, Neurosurgery \& Psychiatry. 2001;71:493-498.

22. Fox CM, Ramig LO, Ciucci MR, Sapir S, McFarland DH, Farley BG, editors. The science and practice of LSVT/LOUD: neural plasticity-principled approach to treating individuals with Parkinson disease and other neurological disorders. Seminars in speech and language; 2006: Copyright $\odot 2006$ by Thieme Medical Publishers, Inc., 333 Seventh Avenue, New York, NY 10001, USA.

23. Ramig L, Countryman S, Thompson L, Horii Y. Comparison of two forms of intensive speech treatment for Parkinson disease. Journal of Speech, Language, and Hearing Research. 1995;38:1232-1251.

24. Ramig L, Halpern A, Spielman J, Fox C, Freeman K. Speech treatment in Parkinson's disease: Randomized controlled trial (RCT). Movement Disorders. 2018;33:1777-1791.

25. Ramig L, Sapir S, Fox C, Countryman S. Changes in vocal loudness following intensive voice treatment (LSVT ${ }^{\circledR}$ ) in individuals with Parkinson's disease: A comparison with untreated patients and normal age-matched controls. Movement Disorders. 2001;16: 79-83.

26. Alharbi GG, Cannito MP, Buder EH, Awan SN. Spectral/Cepstral Analyses of Phonation in Parkinson's Disease before and after Voice Treatment: A Preliminary Study. Folia Phoniatrica et Logopaedica. 2019:1-11.

27. Cannito MP, Suiter DM, Beverly D, Chorna L, Wolf T, Pfeiffer RM. Sentence intelligibility before and after voice treatment in speakers with idiopathic Parkinson's disease. Journal of Voice. 2012;26:214219.

28. Dromey C, Ramig LO, Johnson AB. Phonatory and Articulatory Changes Associated With Increased Vocal Intensity in Parkinson DiseaseA Case Study. Journal of Speech, Language, and Hearing Research. 1995;38:751-764.

29. Fox CM, Morrison CE, Ramig LO, Sapir S. Current perspectives on the Lee Silverman Voice Treatment (LSVT) for individuals with idiopathic Parkinson disease. American Journal of Speech-Language Pathology. 2002;11:111-123.

30. Smith ME, Ramig LO, Dromey C, Perez KS, Samandari R. Intensive voice treatment in Parkinson disease: laryngostroboscopic findings. Journal of Voice. 1995;9:453-459.

31. Ramig L, Dromey C. Aerodynamic mechanisms underlying treatment-related changes in vocal intensity in patients with Parkinson disease. Journal of Speech, Language, and Hearing Research. 1996;39:798-807.

32. Awan SN, Helou LB, Stojadinovic A, Solomon NP. Tracking voice change after thyroidectomy: application of spectral/cepstral analyses. Clinical Linguistics \& Phonetics. 2011;25:302-320.

33. Ma EP-M, Love AL. Electroglottographic evaluation of age and gender effects during sustained phonation and connected speech. Journal of Voice. 2010;24:146-152.

34. Maryn Y, Roy N. Sustained vowels and continuous speech in the auditory-perceptual evaluation of dysphonia severity. Jornal da Sociedade Brasileira de Fonoaudiologia. 2012;24:107-112.

35. Roy N, Mauszycki SC, Merrill RM, Gouse M, Smith ME. Toward improved differential diagnosis of adductor spasmodic dysphonia and muscle tension dysphonia. Folia Phoniatrica et Logopaedica. 2007;59:83-90.

36. Dromey C. Spectral measures and perceptual ratings of hypokinetic dysarthria. Journal of Medical Speech-Language Pathology. 2003;11:85-95.

37. Gerratt BR, Kreiman J, Garellek M. Comparing Measures of Voice Quality From Sustained Phonation and Continuous Speech. Journal of Speech, Language, and Hearing Research. 2016;59:9941001.

38. Watts CR, Awan SN. An examination of variations in the cepstral spectral index of dysphonia across a single breath group in connected speech. Journal of Voice. 2015;29:26-34.

39. Awan SN, Roy N, Jetté ME, Meltzner GS, Hillman RE. Quantifying dysphonia severity using a spectral/cepstral-based acoustic index: comparisons with auditory-perceptual judgements from the CAPE-V. Clinical Linguistics \& Phonetics. 2010;24:742-758. 
40. Halberstam B. Acoustic and perceptual parameters relating to connected speech are more reliable measures of hoarseness than parameters relating to sustained vowels. ORL. 2004;66:70-73.

41. Hillenbrand J. A methodological study of perturbation and additive noise in synthetically generated voice signals. Journal of Speech, Language, and Hearing Research. 1987;30:448-461.

42. Metter EJ, Hanson WR. Clinical and acoustical variability in hypokinetic dysarthria. Journal of Communication Disorders. 1986;19: 347-366.

43. Peterson EA, Roy N, Awan SN, Merrill RM, Banks R, Tanner K. Toward validation of the cepstral spectral index of dysphonia (CSID) as an objective treatment outcomes measure. Journal of Voice. 2013;27:401-410.

44. Awan SN, Roy N, Zhang D, Cohen SM. Validation of the cepstral spectral index of dysphonia (CSID) as a screening tool for voice disorders: development of clinical cutoff scores. Journal of Voice. 2016;30:130-144.

45. Awan SN, Solomon NP, Helou LB, Stojadinovic A. Spectral-cepstral estimation of dysphonia severity: external validation. Annals of Otology, Rhinology \& Laryngology. 2013;122:40-48.

46. Gaskill CS, Awan JA, Watts CR, Awan SN. Acoustic and perceptual classification of within-sample normal, intermittently dysphonic, and consistently dysphonic voice types. Journal of Voice. 2017;31: 218-228.

47. Watts CR, Awan SN. Use of spectral/cepstral analyses for differentiating normal from hypofunctional voices in sustained vowel and continuous speech contexts. Journal of Speech, Language, and Hearing Research. 2011;54:1525-1537.

48. KayPENTAX. Analysis of Dysphonia in Speech and Voice, Model 5109 [Computer program]. Montvale, NJ2011.

49. Awan SN, Roy N. Outcomes measurement in voice disorders: application of an acoustic index of dysphonia severity. Journal of Speech, Language, and Hearing Research. 2009;52:482-499.

50. Awan SN, Roy N, Dromey C. Estimating dysphonia severity in continuous speech: application of a multi-parameter spectral/ cepstral model. Clinical Linguistics \& Phonetics. 2009;23:825-841.

51. Gillespie AI, Dastolfo C, Magid N, Gartner-Schmidt J. Acoustic analysis of four common voice diagnoses: moving toward disorder-specific assessment. Journal of Voice. 2014;28:582-588.

52. Hillenbrand J, Houde RA. Acoustic correlates of breathy vocal quality: dysphonic voices and continuous speech. Journal of Speech, Language, and Hearing Research. 1996;39:311-321.

53. Heman-Ackah YD, Michael DD, Goding GS. The relationship be- tween cepstral peak prominence and selected parameters of dysphonia. Journal of Voice. 2002;16:20-27.

54. Kapoor T, Sharma R. Parkinson's disease diagnosis using Mel-frequency cepstral coefficients and vector quantization. International Journal of Computer Application. 2011;14.

55. Byeon H, Jin H, Cho S. Characteristics of Hypokinetic Dysarthria Patients' Speech based on Sustained Vowel Phonation and Connected Speech. International Journal of u-and e-Service, Science and Technology. 2016;9:417-422.

56. Tjaden K, Sussman JE, Liu G, Wilding G. Long-term average spectral (LTAS) measures of dysarthria and their relationship to perceived severity. Journal of Medical Speech-Language Pathology. 2010;18:125-133.

57. Cannito MP, Suiter DM, Wolf T, Chorna L, Beverly D, Watkins J. Vowel harmonic amplitude differences in a speaker with hypokinetic dysarthria before and after treatment. Journal of Medical Speech-Language Pathology. 2006;14:229-235.

58. Ramig L, Halpern A, Spielman J, Fox C, Freeman K. Speech treatment in Parkinson's disease: Randomized controlled trial (RCT). Movement Disorders. 2018.

59. Fahn S, Elton R. Unified Parkinson's disease rating scale. Fahn S, Marsden CD, Calne D, Goldstein M, editors. Florham Park, NJ: MacMillan Healthcare Information; 1987. p. 153-63.

60. Yorkston KM, Beukelman DR. Assessment of intelligibility of dysarthric speech: Pro-ed; 1984.

61. Fairbanks G. The rainbow passage. Voice and articulation drillbook. 1960;2.

62. Titze IR, Winholtz WS. Effect of microphone type and placement on voice perturbation measurements. Journal of Speech, Language, and Hearing Research. 1993;36:1177-1190.

63. Holm S. A simple sequentially rejective multiple test procedure. Scandinavian Journal of Statistics. 1979:65-70.

64. Cohen J. Statistical power for the behavioural sciences. Hilsdale. NY: Lawrence Erlbaum. 1988.

65. Cohen J. Statistical power analysis for the behavioral sciences 2nd edn. Erlbaum Associates, Hillsdale; 1988.

66. Maryn Y, Roy N, De Bodt M, Van Cauwenberge P, Corthals P. Acoustic measurement of overall voice quality: A meta-analysis a. The Journal of the Acoustical Society of America. 2009;126:26192634.

67. Awan SN, Giovinco A, Owens J. Effects of vocal intensity and vowel type on cepstral analysis of voice. Journal of Voice. 2012;26:670. e15-e20. 